\title{
ESFORÇOS LEGISLATIVOS PARA O CUIDADO DE DOENÇAS RARAS NA CHINA - ANÁLISE DOS FUNDAMENTOS JURÍDICOS E CHAMADA PARA UM QUADRO REGULATÓRIO
}

\author{
Legislative efforts for rare disease in China - Analysis of \\ the legal grounds and call for a regulatory framework
}

Chenguang Wang*

Dingguo Li**

\begin{abstract}
RESUMO
Desde os anos 1980, em resposta aos desafios trazidos pelas doenças raras, alguns países desenvolvidos vêm estabelecendo quadros regulatórios. Os países em desenvolvimento devem fazer o mesmo? Este artigo argumenta que a limitação de recursos de um país em desenvolvimento, embora seja um fator importante para se considerar cuidadosamente a distribuição dos gastos com saúde, não pode ser uma desculpa para negar a necessidade de uma regulamentação. $O$ trabalho apresenta argumentos legais e políticos para o estabelecimento de tal regulação nos países em desenvolvimento. Também relata os recentes esforços legislativos para o cuidado das doenças raras por parte das autoridades locais chinesas, explica as razões para a adoção de uma nova definição de doenças raras e elabora os segmentos necessários dentro de um contexto integrado, racional e adequado à China para o cuidado dessas enfermidades.
\end{abstract}

Palavras-chave: China; Direitos Humanos; Doenças Raras; Justiça; Quadro Regulatório.

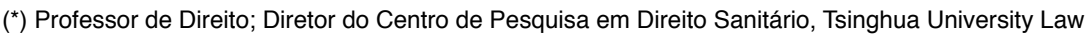
School. Pequim - China. E-mail: wcglaw@tsinghua.edu.cn.

$\left.{ }^{* *}\right)$ Professor de Medicina; Diretor da Escola de Graduação, Shanghai Second Medical University. Vice-reitor, Pediatrics Medical College e Xinhua Medical College. Diretor de Gastroenterologia, Xinhua Hospital. Membro da Conferência Consultiva Política Popular Chinesa. Chinese People's Political Consultative Conference. ; Vice-secretário da Conferência Consultiva Política Popular de Changhai. People's Political consultative Conference. Pequim - China.

Artigo recebido em: 07/09/2012. Revisado em: 17/10/2012. Aprovado em: 21/10/2012. 


\begin{abstract}
In response to the challenges posed by rare diseases, some developed countries have set up regulatory frameworks since 1980s. Should developing countries follow suit? This article argues that the limited resources of a developing country, although a major factor for carefully considering just distribution of health spending, should not be used as an excuse to reject the need for such a regulatory framework. It provides both policy and legal arguments for establishing such a framework in developing countries. It also reports the recent development of rare disease legislative efforts by local authorities in China, explains the reasons of a new definition of rare diseases adopted, and elaborates the necessary blocks in an integrated rational framework for rare diseases suitable for China.
\end{abstract}

Keywords: China; Justice; Human Rights; Rare Diseases, Regulatory Framework.

\title{
I. A especificidade das doenças raras traz novos desafios e pede um novo quadro regulatório
}

O termo "doenças raras", como o próprio nome diz, refere-se a enfermidades com relativa baixa incidência, que implicam desafios à ciência médica e criam barreiras aos pacientes que buscam desesperadamente tratamento médico. As doenças raras não apenas infringem dores enormes e sofrimento aos doentes e seus familiares, ${ }^{(1)}$ como também impactam negativamente em toda a sociedade e dificultam o desenvolvimento social e a realização individual.

De uma maneira geral, a legislação, as políticas públicas e os mecanismos regulatórios em um país são elaborados para responder a temas convencionais e problemas de rotina que surgem no dia a dia. No entanto, as doenças raras são casos excepcionais e, como tais, representam novos, embora frequentemente negligenciados, desafios à ciência médica e à indústria farmacêutica, como, por exemplo, a falta de informação e de conhecimento por parte da sociedade e até mesmo por parte dos profissionais médicos; sistemas de diagnóstico deficientes; altos custos; ${ }^{(2)}$ dificuldades em desenvolver e testar novas drogas em razão de sua baixa frequência e de retornos mercadológicos incertos. Além disso, as doenças raras também colocam novos desafios para toda a sociedade, como o fardo financeiro sobre os pacientes e suas famílias em razão dos altos preços dos medicamentos; ${ }^{(3)}$ significativo estresse psicológico pelo isolamento social;

\footnotetext{
1 JIE, Zhao; QIAOWEN, Xu et al. China has more than ten million rare disease patients and the majority are dying without affordable drugs. News Report by Guangzhou Daily, March 21, 2011.

2 STOLK, P.; WILLEMEN, M.J.; LEUFKENS, H.G. Rare essentials: drugs for rare diseases as essential medicines. Bull World Health Organ, n. 84, p. 746, 2006

${ }^{3}$ Id. Ibid.
} 
desemprego e incertezas quanto ao futuro;(4) o estigma associado a esse tipo de enfermidade e a limitação da cobertura de seguros. Todas essas questões são muito modernas para as tradicionais regulações legais, o que significa que os sistemas existentes não podem lidar com esses desafios de maneira eficiente. A baixa frequência dessas doenças contrasta de maneira gritante com a prevalência das enfermidades comuns e, por esse motivo, demanda novos instrumentos legais e um quadro regulatório especial. Nesse sentido, a criação de instrumentos legais apropriados, tanto em nível nacional como internacional, para lidar com a excepcionalidade das doenças raras é uma necessidade sentida não apenas pelos doentes e seus familiares, como também pelos profissionais da saúde e cidadãos. Esses novos instrumentos e quadro regulatório devem ser distintos dos sistemas convencionais, em razão da natureza excepcional das questões levantadas pelas doenças raras.

"Em resposta a isso, a primeira legislação sobre droga órfã foi introduzida nos Estados Unidos em 1983. Outros países (como a Austrália, Japão e Cingapura) seguiram o exemplo nos anos 1990 e, em 2000, foi a vez de a União Europeia estabelecer uma legislação própria sobre o assunto". (5) "De fato (na Europa), durante as duas últimas décadas, o reconhecimento público de que as doenças raras são um tema importante do ponto de vista médico e social vem crescendo gradativamente na consciência pública, resultado do trabalho intenso de advocacy por parte de grupos que incluem acadêmicos (pesquisadores das ciências básicas e médicas) e políticos". As doenças raras se tornaram "uma questão médica e social importante". ${ }^{(6)}$

\section{Os países em desenvolvimento também devem ter um quadro regulatório sobre 0 assunto?}

Em contraste, na maioria dos países em desenvolvimento não existe um quadro regulatório para o cuidado das doenças raras. Os defensores da não necessidade de uma regulamentação sobre o assunto afirmam que as preocupações primeiras devem focar em temas relacionados à maioria da população, como a pobreza, habitação, atenção médica primária etc. Por conseguinte, os governos dos países em desenvolvimento não contam com recursos suficientes, conhecimento e capacidade para lidar com as doenças raras. Como resultado, a necessidade de lidar com temas convencionais muitas vezes ofusca a demanda e a legitimidade de um quadro regulamentador para essas enfermidades. A pergunta que

\footnotetext{
${ }^{4}$ ZURYNSKI, Y.; FRITH, K.; LEONARD, H.; ELLIOTT, E. Rare childhood diseases: how should we respond? Arch Dis Child n. 93, p. 1071-1074, 2008. Originariamente publicado online em 6 de agosto de 2008. Disponível em: <http://www.adc.bmj.com>. Acesso em: 3 mar. 2012.

5 STOLK, P.; WILLEMEN, M.J.; LEUFKENS, H.G. op. cit.

${ }^{6}$ SCHIEPPATI, Arrigo; HENTER, Jan-Inge; DAINA, Erica; APERIA, Anita. Why rare diseases are an important medical and social issue? The Lancet, n. 371, p. 2039-2041, 2008. Disponível em: <http:// www.myelin.org/en/art/34/>. Acesso em: 25 jan. 2012.
} 
se faz é a seguinte: os países em desenvolvimento devem estabelecer essa regulamentação? Um pequeno grupo de pessoas (pacientes com enfermidades raras) deve se manifestar em relação à divisão desproporcional dos limitados recursos nacionais com base em um conceito de justiça distributiva?

Não se podem negar algumas verdades, como o fato de que o quadro regulatório para o cuidado das doenças raras depende amplamente da disponibilidade de recursos. As políticas sociais e a legislação de um país não podem estar deslocadas de seu contexto social, especialmente em relação aos recursos disponíveis. Reconhecendo totalmente que os recursos sociais limitados são grandes restrições para gastos extras com o tratamento das doenças raras, este artigo argumenta que, desde que esses recursos são sempre relativamente limitados, sua insuficiência não pode ser usada como uma razão convincente para se ignorar por completo a existência das enfermidades raras e para impedir o avanço e mesmo lutar contra um quadro regulatório de atenção a elas. De fato, algumas dessas doenças não requerem grandes investimentos governamentais, uma vez que a prevenção, o diagnóstico e tratamento precoces são menos custosos e mais eficientes. Além disso, o desenvolvimento de drogas órfãs pode estimular "novos caminhos de investimentos para a indústria farmacêutica". ${ }^{(7)}$ Pesquisas sobre enfermidades raras podem contribuir para o avanço da medicina ao prevenirem futuros problemas de saúde pública, encontrarem meios de cura e de controle de síndromes e produzirem mais conhecimento sobre a biologia humana. ${ }^{(8)}$ Essas pesquisas também colaboram para a promoção da saúde pública e para a melhoria da qualidade de vida da coletividade. A alocação de recursos para as doenças raras pode ser limitada, mas, ainda assim, pode ajudar alguns pacientes, demonstrando uma orientação humana das políticas públicas e conquistando um objetivo social mais valioso.

Este artigo propõe três argumentos básicos como base para um novo quadro regulatório legal voltado para o cuidado das doenças raras. Primeiro: tal regulação irá promover futuras descobertas científicas e desenvolvimento da área médica e da indústria biofarmacêutica. É verdade que uma nova regulação irá consumir recursos. No entanto, ao considerar os gastos com essas enfermidades, o governo deve considerar também a melhora da vida dos pacientes e de suas famílias e o desenvolvimento social. Nesse sentido, devem ser adotados um plano de longo prazo e uma visão mais abrangente; não se deve olhar apenas para os retornos econômicos e comerciais, mas considerar ainda o desenvolvimento social e humano alcançado.

Em segundo lugar, distribuir recursos de maneira imparcial não significa simplesmente alocá-los uniformemente. "Equidade significa justiça social" e

SCHIEPPATI, Arrigo; HENTER, Jan-Inge; DAINA, Erica; APERIA, Anita. op. cit.

8 DODGE, John A; CHIGLADZE, Tamara; DONADIEU, Jean, et al. The importance of rare diseases: from the gene to society. Arch Dis Child, n. 96, p. 791-792, 2011. Originariamente publicado online em 12 de agosto de 2010. Disponível em: <http://www.adc.bmj.com>. Acesso em: 3 mar. 2012. 
"Igualdade não é o mesmo que equidade".(9) Nesse sentido, a legislação para as doenças raras envolve a questão filosófica de redistribuição de recursos como forma de promover a justiça social. A distribuição de recursos tem como base o conceito da justiça distributiva defendido por Aristóteles, bem diferente do conceito moderno, ${ }^{(10)}$ fundamento filosófico e político do sistema de bem-estar social contemporâneo. O novo conceito de justiça distributiva evidencia a equidade em lugar da igualdade na alocação de recursos entre os diferentes grupos sociais, particularmente em favor dos grupos mais vulneráveis, a fim de manter níveis básicos de qualidade de vida. Algumas enfermidades raras não requerem drogas ou tratamentos caros, e diagnóstico, intervenções e tratamentos precoces resultam em custos mais baixos. Nesse sentido, não é justo virar as costas aos pacientes com doenças raras, apenas porque sua enfermidade se encaixa na categoria de "rara". A doutrina da justiça distributiva justifica a inclusão de drogas órfãs na lista de medicamentos essenciais ${ }^{(11)}$ e a oferta de cobertura para doentes com enfermidades raras.

Por fim, um terceiro ponto trata da vida humana e da saúde, que são consideradas universalmente os valores mais sagrados. "O direito à saúde conforme estabelecido na Constituição da Organização Mundial da Saúde e nos tratados internacionais de direitos humanos é o direito ao 'mais alto nível possível de saúde'". ${ }^{(12)}$ Com base nesse julgamento moral e legal, um quadro regulatório para o tratamento das doenças raras conta com uma base legal sólida e justificada.

Para os países desenvolvidos com um relativo progresso econômico, como China e México, a necessidade de um quadro regulatório para o cuidado das doenças raras deve ser reconhecida e estabelecida sem atraso. O desenvolvimento econômico produz um bolo maior de receitas e prepara o terreno para tal sistema regulatório. Enquanto a expansão do sistema de bem-estar social pressiona para maior cobertura além dos convencionais cuidados de atenção primária à saúde e o senso de justiça social estimula maior proteção aos grupos vulneráveis, um marco regulatório para o cuidado das doenças raras deve ser incluído no sistema legal. Em anos recentes, com o aumento da preocupação da sociedade com as enfermidades raras, na China, foram feitos esforços pressionando o governo a definir essa política regulatória. ${ }^{(13)}$ Fica muito claro, entretanto, que "decisões não podem ser tomadas apenas com base na economia e devem

\footnotetext{
9 BRAVEMAN, P.; GRUSKIN, S. Defining equity in health. Journal of Epidemiol Community Health, v. 57, n. 4 , 2003. Neste artigo, eles afirmam: "O conceito de equidade é inerentemente normativo, quer dizer, com base em valores, enquanto o de igualdade não é necessariamente assim".

${ }^{10}$ FLEISCHACKER, Samuel. A short history of distributive justice. Harvard: Harvard University Press, 2005. p. 13.

${ }^{11}$ REIDENBERG, Marcus M. Are drugs for rare diseases "essential"? Bulletin of the World Health Organization, v. 84, n. 9, Sept. 2006.

${ }^{12}$ BRAVEMAN, P.; GRUSKIN, S. op. cit.

${ }^{13}$ WANG, Jing-Bo; GUO, Jeff J.; YANG, Li; ZHANG, Yan-De; SUN, Zhao-Qi; ZHANG, Yan-Jun. Rare diseases and legislation in China. The Lancet, v. 375, n. 9716, p. 708-709, Feb. 27, 2010.
} 
ser consideradas dentro de um contexto que evidencia a pesquisa médica e os princípios morais e éticos."(14)

\section{Bases legais para um quadro regulatório para o tratamento das doenças raras na China}

Como pré-requisito, toda política pública adequada e legislação devem ter bases legítimas. Quais são as bases legitimadoras para uma legislação e políticas públicas voltadas para o cuidado de enfermidades raras na China?

Doenças raras não são apenas questões médicas, mas temas sociais relacionados aos direitos humanos básicos dos pacientes. $O$ direito à saúde é um direito legalmente reconhecido na China. O Art. 33 da Constituição da República Popular (CRP) da China estipula que "o Estado respeita e protege direitos humanos." O direito à saúde é o mais fundamental deles. Segundo René Descartes, "a preservação da saúde é (...) sem dúvida a primeira riqueza e o fundamento de todas as outras riquezas na vida”. ${ }^{(15)}$ A saúde é considerada tão importante porque (a) ela é diretamente constitutiva do bem-estar da pessoa e (b) ela permite que a pessoa atue como agente, quer dizer, que ela persiga metas e projetos de vida que valoriza. ${ }^{(16)}$ No processo de construção de uma sociedade harmônica com base no princípio de "pessoas em primeiro lugar", as políticas públicas e os dispositivos legais não podem ignorar a questão das doenças raras simplesmente por causa de sua raridade e baixa prevalência.

O Art. 21 da CRP afirma que "O Estado é responsável pelos serviços médicos e de saúde (...) para proteger a saúde das pessoas”. O Art. 45 vai além ao afirmar: "Cidadãos idosos, doentes ou deficientes da República Popular da China têm o direito à assistência material do Estado e da sociedade. O Estado é responsável pelos serviços de segurança social, assistência social e serviços médicos e de saúde necessários para os cidadãos desfrutarem desse direito". Esse artigo impõe uma obrigação legal aos governos e confere um direito aos cidadãos. Ele oferece as bases constitucionais para a formulação de políticas públicas e instrumentos legais para lidar com as doenças raras na China.

Junto com o desenvolvimento social e econômico, a China conta com mais recursos e condições materiais para oferecer maior assistência e proteção aos pacientes com doenças raras, assim como para promover pesquisa médica e o segmento da indústria farmacêutica. Para alcançar justiça social, é imperativo oferecer mais assistência afirmativa e proteção aos doentes com enfermidades raras.

\footnotetext{
${ }^{14}$ ZURYNSKI, Y.; FRITH, K.; LEONARD, H.; ELLIOTT, E. op. cit.

${ }^{15}$ DESCARTES, René. Méthode published in 1637, cited from Author Affiliations, The Concern for Equity in Health. J Epidemiol Community Health, 2002. Disponível em: <http://jech.bmj.com/ content/56/7/485.full>. Acesso em: 26 jan 2012.

${ }^{16}$ Id. Ibid.
} 
Com base nas análises apresentadas, este artigo propõe os seguintes princípios gerais para a formulação de políticas públicas e a elaboração de regulações para o tratamento das doenças raras: (1) os princípios de proteção aos direitos humanos e de "pessoas em primeiro lugar"; (2) os princípios da justiça distributiva e da equidade com ações afirmativas para os grupos vulneráveis; (3) os princípios de buscar a verdade dos fatos e de uma abordagem progressiva e científica no processo de elaboração das leis; (4) os princípios de visão abrangente para coordenar esforços privados e públicos no gerenciamento das doenças raras, buscando a promoção dos segmentos da indústria das drogas órfãs e da pesquisa científica, a disponibilização de serviços médicos adequados e cobertura médica e a implantação de redes nacionais de informação, educação e de especialistas; e (5) o princípio de enfatizar diagnósticos precoces, prevenção oportuna e tratamento efetivo.

\section{Definição proposta de doenças raras e o quadro regulatório na China}

Em 17 de maio de 2010, um grupo de especialistas das áreas da medicina, sociologia, direito e economia reuniram-se em Xangai para discutir como construir um quadro regulatório para doenças raras. Percebendo que seria difícil elaborar uma legislação sobre doenças raras em nível nacional, pela falta de compreensão sobre essas enfermidades e pela falta de experiência em lidar com elas, os especialistas concluíram que os governos locais e das províncias, como Xangai, deveriam tomar a iniciativa de definir uma legislação própria. ${ }^{(17)}$

A definição de doenças raras é a primeira questão legal a ser enfrentada. Até agora não há uma definição legal para doenças raras aceita internacionalmente, mas existem definições bastante específicas de cada país, derivadas de seus respectivos contextos sociais.

O decreto norte-americano sobre doenças raras de 2002 definiu essas enfermidades com base em sua prevalência. Qualquer condição que afete menos do que 200 mil pessoas no país (quase 1 em 1.500) foi considerada uma doença rara; nada muito diferente da definição encontrada no decreto sobre drogas órfãs de 1983 cujo objetivo era encorajar as pesquisas sobre essas enfermidades e suas possíveis curas. Os japoneses definiram como doença órfã (...) aquela que afeta menos do que 50 mil pessoas (1 a cada 2.500). Na Europa, por outro lado, essas enfermidades são determinadas, não pela prevalência, mas pelo risco de vida que elas causam ou pela sua capacidade de debilitar cronicamente uma pessoa e pelo esforço necessário para combatê-las. Em

\footnotetext{
${ }^{17}$ Esse esforço tem resultado em respostas positivas dos governos de Xhangai, Guangzhou e Pequim. Shangai em particular iniciou um estudo sobre legislação em 2011 e os outros dois governos receberam propostas de uma legislação para doenças raras.
} 
virtude dessa definição, o limite de prevalência para a maioria dessas doenças era de 1 em 2.000, um meio termo entre Estados Unidos e Japão. ${ }^{(18)}$

A OMS sugere uma frequência menor do que 6,5 a 10 a cada 10 mil pessoas. ${ }^{(19)}$ Até agora, a China não tem regulações específicas para tratar das doenças raras, muito menos uma definição legal. O que fica claro é que não importa o conceito internacional ou de outro país, pois os pacientes atingidos por doenças raras na China constituem-se em um grande grupo de pessoas em função da enorme população nacional.

Considerando a situação demográfica e econômica da China, os especialistas foram muito cautelosos e realísticos ao proporem que uma doença rara é aquela que afeta menos do que uma pessoa a cada 500 mil e menos do que uma criança a cada 10 mil, como as doenças genéticas raras. Com o objetivo de oferecer tratamento adequado e efetivo, eles também propuseram uma lista de 23 enfermidades como um primeiro grupo de doenças raras. ${ }^{(20)}$

As razões para tais proposições são: em primeiro lugar, os padrões formulados em outros países não se adequam ao fenômeno da imensa população chinesa. Uma proporção sensata deve ser elaborada com base na realidade econômica e demográfica. Em segundo lugar, uma vez que as doenças genéticas compreendem mais de $80 \%$ das doenças raras e que $50 \%$ das enfermidades genéticas afetam as crianças, ${ }^{(21)}$ é mais prático e factível começar com as doenças raras genéticas, fornecendo diagnóstico precoce e tratamento efetivo de maneira sistemática para os recém-nascidos. $\mathrm{E}$, em terceiro lugar, uma vez que existam "entre seis mil e oito mil doenças raras" e que "quase sempre não há curas definitivas" para a maioria delas, ${ }^{(22)}$ é razoável listar 23 enfermidades que são passíveis de serem curadas pela medicina atual e que são cobertas pela capacidade financeira governamental. Ou seja, o primeiro grupo de pacientes com doenças raras a se qualificar para a cobertura de seguro de saúde e tratamento médico não deve ser tão grande a ponto de espantar os governos e outras organizações; e as doenças raras assim definidas devem ser possíveis de serem curadas pela medicina ou tratamentos disponíveis. Como resultado dessa definição, o número total de pacientes com doenças raras reconhecidas pela proposição é menor do que $1 \%$ do número total de pacientes com doenças

\footnotetext{
${ }^{18}$ BRADLEY, David. Rare diseases and rarer treatments. Disponível em: <http://www.sciencebase. com/science-blog/rare-diseases-and-rarer-treatments.html>. Acesso em: 25 jan. 2012.

${ }^{19}$ ARONSON, J. K. Rare diseases and orphan drugs. Disponível em: <http://onlinelibrary.wiley.com/ doi/10.1111/j.1365-2125.2006.02617.x/full>. Acesso em: 26 jan. 2012.

${ }^{20}$ MINUTES of Experts Meeting on Rare Diseases in Shanghai, May 17, 2010. What is a rare disease? Disponível em: <http://www.rarediseaseday.org/article/what-is-a-rare-disease>. Acesso em: 26 jan. 2012.

${ }^{21}$ MINUTES of Experts Meeting on Rare Diseases in Shanghai, May 17, 2010. What is a rare disease?, cit.

${ }^{22}$ Supra.
} 
raras na China. Os especialistas também sugeriram que devem ser elaboradas definições correspondentes para drogas órfãs, políticas públicas locais e regulações para sua pesquisa, produção e propaganda. Essa sugestão recebeu uma resposta positiva dos governos locais como o de Xanghai.

Essa definição foi oficialmente incorporada na Notificação para Inclusão de Drogas Especiais para Doenças Raras na Cobertura de Fundos Mútuos para a Infância de Xangai (Notificação 39), pelo Gabinete de Gestão de Fundos Mútuos de Internação de Crianças e Alunos de Escolas Fundamentais e Básicas, em 29 de agosto de 2011. ${ }^{(23)}$ Foram relacionadas apenas quatro doenças ${ }^{(24)}$ a serem tratadas em três hospitais em Xhangai. ${ }^{(25)}$ Apesar de ser um pequeno passo, ele começou a preencher o vácuo existente pela falta de um quadro legal na China. O ministro da Saúde, Chen Zhu, recentemente afirmou que, em relação às drogas órfãs, o Estado deve adotar produção uniformizada em lugares designados e processos uniformizados de licitação, compra, distribuição e armazenagem com o objetivo de garantir seu fornecimento. ${ }^{(26)}$

Com base nas políticas públicas e regulações mencionadas, a proposição de um quadro regulatório deve considerar a necessidade de um planejamento de abrangência nacional e de esforços conjuntos, para coordenar ações dos setores público e privado e, em lugar da segmentação, associar todas as áreas envolvidas, desde a indústria até a pesquisa médica, cobertura de saúde e educação pública. Essa proposição deve ainda incluir os seguintes blocos, cada qual atuando como um subsistema correspondente. ${ }^{(27)}$

1. Serviços de saúde de qualidade que ofereçam acesso aos serviços médicos e tratamentos, de maneira efetiva e adequada. Eles devem incluir: (a) diagnóstico precoce em recém-nascidos; (b) tratamento e reabilitação; (c) redes funcionais de especialistas e plataformas de assistência médica a longa distância; (d) centros nacionais e regionais de bancos de dados e de troca de informações; e (e) redes educacionais para treinamento profissional e educação pública.

2. Um segmento privado de drogas órfãs que funcione bem e que inclua: (a) uma indústria dinâmica com áreas de pesquisa, produção e distribuição;

\footnotetext{
${ }^{23}$ NOTIFICATION on Including Special Drugs for Rare Diseases into the Coverage of Shanghai Mutual Funds for Infants and Children, N. 38, [2011], by Hu-Er-Ji-Jin-Ban (Management Office of Mutual Funds for Hospitalization of Infants, Elementary and Secondary School Pupils) on Aug. 29, 2011.

${ }^{24}$ As quatro doenças relacionadas são: Doença de Pompe, Doença de Gaucher, Doença de Fabry e Mucopolissacaridose.

${ }^{25}$ Os três hospitais designados para tratamento de doenças raras são Xinhua Hospital, ligado à Jiaotong University, Hospital Pediátrico da Fudan University e Ruijin Hospital, também ligado à Jiaotong University.

${ }^{26}$ ZHIMEI, Fu. The State Should Guarantee Supply of Orphan Drugs by Uniform Production, Purchase, Distribution and Restoration. People's Daily, Dec. 21, p. 13, 2011.

${ }^{27}$ CHENGUANG, Wang. To establish a suitable rare diseases mechanism in China. Chinanews, Special Issue on Rare Diseases Prevention and Cure, p. 19, Mar. 2011.
} 
(b) gerenciamento eficiente por parte de órgãos governamentais que inclua testes, aprovação, registro e controle de segurança; (c) financiamento adequado para a indústria; (d) aprovação rápida para registros estrangeiros de drogas órfãs e para sua distribuição internacional; e (e) incentivos efetivos para a produção de drogas órfãs e para sua distribuição, como instrumentos legais de proteção da propriedade intelectual, monopólio exclusivo do mercado, benefícios fiscais, suporte financeiro, redes comerciais etc.

3. Seguro médico e social adequado para tornar os cuidados médicos e medicamentos mais acessíveis aos pacientes, aliviando a carga sobre eles e suas famílias e proporcionando-Ihes condições básicas de vida. Esse seguro é formado pelas seguintes partes: (a) controle de preços, por um lado, para oferecer serviços e drogas acessíveis, seja pelo controle de preços, seja por meio de subsídios para os pacientes; por outro lado, para permitir que os pesquisadores produtores e distribuidores de medicamentos órfãos possam ter retorno financeiro adequado; (b) dispositivos para incluir drogas órfãs na lista de medicamentos essenciais; (c) seguro de saúde e assistência social: deve haver razões legais para obrigar as empresas de seguros a oferecer a cobertura de saúde para os pacientes com doenças raras; devem ser estabelecidos incentivos e procedimentos adequados para a mobilização de organizações sociais e de caridade para prestar assistência financeira e profissional a pacientes carentes, como os pobres e deficientes; e (d) redes de assistência mútua, as quais devem incentivar instituições como as associações de pacientes, organizações não governamentais e associações profissionais de assistência.

4. Institutos de pesquisa e plataformas de educação pública. Os efeitos desses instrumentos são o de promover a sensibilização do público para as doenças raras, disseminar o conhecimento sobre elas, eliminar o preconceito social contra pacientes e estabelecer uma plataforma pública de troca de informações.

5. Coordenação e gestão eficiente. As doenças raras são incomuns e sua incidência se distribui de maneira ampla. Portanto, o acesso à assistência médica eficaz e a medicamentos apropriados é frequentemente bloqueado ou atrasado. Em tal contexto social, gestão e coordenação integrada são cruciais para lidar com essas doenças. Aqui incluem-se: (a) gabinetes de coordenação de drogas e equipamentos órfãos em níveis nacional e locais responsáveis pela elaboração de normas, promoção de pesquisas, produção e distribuição de drogas e equipamentos órfãos; (b) coordenação em ambos os níveis nacional e locais para o tratamento de doenças raras, responsáveis pela criação de redes de especialistas, plataformas de informação, centros de treinamento de longa distância para profissionais dos serviços médicos; (c) coordenadorias regionais do seguro social para o gerenciamento inter-região de seguro médico e social nacional; (d) organizações de pacientes com doenças raras que forneçam informações e assistência em relação aos 
medicamentos órfãos, tratamento, recuperação e reabilitação; e (e) órgãos de cooperação internacional e externos responsáveis pela colaboração e compartilhamento de informações em níveis regionais e internacional etc.

Esses segmentos e as partes que os compõem são interdependentes e juntos eles formam um quadro regulatório integrado e abrangente. Os governos devem utilizar os esforços conjuntos de ambos os setores público e privado para arcar com essa obrigação legal e moral e assumir a liderança na formação de tal marco.

Doenças raras trazem novos desafios para a sociedade, em particular para os países em desenvolvimento. O sucesso ao lidar com esses desafios depende de nossa capacidade em fazer inovações sistêmicas e desenvolver um novo quadro regulatório. Nesse sentido, o significado do quadro proposto para o gerenciamento das doenças raras ultrapassa o âmbito da ciência médica e alcança o campo da inovação institucional e reforma social.

\section{Referências}

ARONSON, J. K. Rare diseases and orphan drugs. Disponível em: <http:// onlinelibrary.wiley.com/doi/10.1111/j.1365-2125.2006.02617.x/full>. Acesso em: 26 jan. 2012.

BRADLEY, David. Rare diseases and rarer treatments. Disponível em: <http:// www.sciencebase.com/science-blog/rare-diseases-and-rarer-treatments.html>. Acesso em: 25 jan. 2012.

BRAVEMAN, P.; GRUSKIN, S. Defining equity in health. Journal of Epidemiol Community Health, v. 57, n. 4, 2003.

CHENGUANG, Wang. To establish a suitable rare diseases mechanism in China. Chinanews, Special Issue on Rare Diseases Prevention and Cure, Mar. 2011.

DESCARTES, René. Méthode published in 1637, cited from Author Affiliations, The Concern for Equity in Health. J Epidemiol Community Health, 2002. Disponível em: <http://jech.bmj.com/content/56/7/485.full>. Acesso em: 26 jan. 2012.

DODGE, John A; CHIGLADZE, Tamara; DONADIEU, Jean, et al. The importance of rare diseases: from the gene to society. Arch Dis Child, n. 96, p. 791-792, 2011. Originariamente publicado online em 12 de agosto de 2010. Disponível em: <http://www.adc.bmj.com>. Acesso em: 3 mar. 2012.

FLEISCHACKER, Samuel. A short history of distributive justice. Harvard: Harvard University Press, 2005.

JIE, Zhao; QIAOWEN, Xu et al. China has more than ten million rare disease patients and the majority are dying without affordable drugs. News Report by Guangzhou Daily, March 21, 2011. 
MINUTES of Experts Meeting on Rare Diseases in Shanghai, May 17, 2010. What is a rare disease? Disponível em: <http://www.rarediseaseday.org/article/ what-is-a-rare-disease>. Acesso em: 26 jan. 2012.

NOTIFICATION on Including Special Drugs for Rare Diseases into the Coverage of Shanghai Mutual Funds for Infants and Children, N. 38, [2011], by Hu-ErJi-Jin-Ban (Management Office of Mutual Funds for Hospitalization of Infants, Elementary and Secondary School Pupils) on Aug. 29, 2011.

REIDENBERG, Marcus M. Are drugs for rare diseases "essential"? Bulletin of the World Health Organization, v. 84, n. 9, Sept. 2006.

SCHIEPPATI, Arrigo; HENTER, Jan-Inge; DAINA, Erica; APERIA, Anita. Why rare diseases are an important medical and social issue? The Lancet, n. 371, p. 2039-2041, 2008. Disponível em: <http://www.myelin.org/en/art/34/>. Acesso em: 25 jan. 2012.

STOLK, P.; WILLEMEN, M.J.; LEUFKENS, H.G. Rare essentials: drugs for rare diseases as essential medicines. Bull World Health Organ, n. 84, p. 745-751, 2006.

WANG, Jing-Bo; GUO, Jeff J.; YANG, Li.; ZHANG, Yan-De.; SUN, Zhao-Qi; ZHANG, Yan-Jun. Rare diseases and legislation in China. The Lancet, v. 375, n. 9716, p. 708-709, Feb. 27, 2010.

ZHIMEI, Fu. The State Should Guarantee Supply of Orphan Drugs by Uniform Production, Purchase, Distribution and Restoration. People's Daily, Dec. 21, 2011.

ZURYNSKI, Y.; FRITH, K.; LEONARD, H.; ELLIOTT, E. Rare childhood diseases: how should we respond? Arch Dis Child n.93, p.1071-1074, 2008. Originariamente publicado online em 6 de agosto de 2008. Disponível em: <http:// www.adc.bmj.com>. Acesso em: 3 mar. 2012.

Agradecimentos ao Sr. Seth Ure pela edição deste manuscrito. 